\title{
Evaluación del aprendizaje basado en problemas y examen final: diferencias en la percepción de justicia y el desarrollo de competencias Problem-based learning assessment and final examination: differences in the perception of justice and the competencies development
}

\author{
Ana M. Castaño ${ }^{1}$, Pedro J. Ramos-Villagrasa ${ }^{2}$, Antonio L. García Izquierdo ${ }^{1}$ \\ castanoana@uniovi.es,pjramos@unizar.es, angarcia@uniovi.es \\ ${ }^{1}$ Departamento de Psicología \\ Universidad de Oviedo \\ Oviedo, España \\ ${ }^{2}$ Departamento de Psicología y Sociología \\ Universidad de Zaragoza \\ Zaragoza, España
}

\begin{abstract}
Resumen- El examen es uno de los métodos de evaluación mayoritariamente utilizados en el contexto universitario. No obstante, su conexión con la capacidad para el afrontamiento y resolución de los problemas significativos de la vida profesional futura del alumnado ha sido cuestionada, y la evaluación del aprendizaje basado en problemas cobra cada vez más relevancia como alternativa al examen. Esta evaluación se centra en situaciones de aprendizaje de la vida real y puede suponer un beneficio tanto para el alumnado como para el marco de enseñanza actual, que demanda procesos de evaluación justos y que permitan al alumnado el desarrollo de competencias útiles para su desempeño laboral futuro. Con el objetivo de comprobar si la evaluación del aprendizaje basado en problemas supone una mejora de la percepción de justicia procedimental y la percepción del desarrollo de competencias del alumnado, 44 estudiantes universitarios fueron evaluados mediante ambos métodos y posteriormente cumplimentaron un cuestionario para su comparación. Los resultados pusieron de manifiesto los beneficios de la evaluación del aprendizaje basado en problemas.
\end{abstract}

\section{Palabras clave: evaluación, justicia, competencias}

Abstract- The final examination is one of the assessment methods mostly used in the university context. However, their relatedness with the ability for coping and resolving significant problems of the future professional life of the students have been questioned, and problembased learning assessment becomes increasingly relevant as an alternative to exam. This assessment is focused on real-life learning situations and may involve a benefit to both students and the current educational framework, which requires fair evaluation processes that allow students to develop useful competencies for their future work performance. In order to test if the problem-based learning system implies an improvement in the perception of procedural justice and in the perception of students' development of competences, 44 university students were evaluated by means of both methods and then completed a questionnaire to compare them. The results highlighted the benefits of the problem-based learning assessment.

Keywords: assessment, justice, competencies

\section{INTRODUCCIÓN}

La evaluación es una de las actividades de mayor relevancia en el ámbito educativo, pero esta ha estado tradicionalmente centrada en una función sumativa mediante un examen final, ignorando su papel en la mejora de los aprendizajes del alumnado (Gil y Padilla, 2009). El nuevo contexto del Espacio Europeo de Educación Superior requiere un enfoque docente basado en competencias en el que el alumnado esté situado en el centro del proceso de aprendizaje (Delgado y Oliver, 2006) y que le prepare para el afrontamiento y resolución de los problemas significativos de la vida profesional futura.

Asimismo, este nuevo contexto requiere unos procesos de evaluación que garanticen el principio de justicia, dado que las instituciones educativas tienen una finalidad social y pueden tener un importante impacto en la vida futura del alumnado (Niessen, Meijer, y Tendeiro, 2017). Diversas investigaciones han puesto de relevancia el potencial de la justicia organizacional para generar beneficios en las organizaciones y sus empleados (i.e. Colquitt, Conlon, Wesson, Porter, y Ng, 2001). En concreto, la justicia procedimental (Cohen-Charash y Spector, 2001) hace referencia a la justicia percibida en los procedimientos utilizados para tomar decisiones, y aunque ha sido principalmente estudiada en contextos organizativos, sus principios y beneficios se pueden trasladar al proceso de evaluación en el ámbito educativo partiendo de la teoría organizacional de Gilliland (1993). En este sentido la evaluación universitaria debería ser justa y permitir al alumno conocer qué debe mejorar para enfrentarse a situaciones de aprendizaje futuras (Zaragoza, Luis-Pascual, y Manrique, 2009).

Ante estas demandas, los sistemas de evaluación que se centran en situaciones de aprendizaje de la vida real y en problemas significativos que requieren el uso de conocimientos, habilidades y actitudes que van más allá de los exámenes tradicionales (Coll, Rochera, Mayordomo, y Naranjo, 2007) emergen como alternativas al examen. En concreto, uno de estos sistemas de evaluación alternativos es la evaluación del aprendizaje basado en problemas (Barrows, 1986). En este tipo de aprendizaje se plantea un problema al que el alumnado debe dar respuesta, principalmente mediante 
el trabajo colaborativo en grupo, y en el que el profesorado actúa como facilitador de los procesos y avances, sirviendo de guía y orientación. Siguiendo a Solaz-Portolés, Sanjosé y Gómez (2011) algunas de las características y también ventajas destacables del aprendizaje basado en problemas son: (i) utiliza una metodología de trabajo que desarrolla habilidades cognitivas de alto nivel, (ii) promueve la metacognición y su aprendizaje autorregulado, (iii) sitúa al alumnado en un papel de profesionales que intentan resolver un problema o situación próxima a la realidad, (iv) requiere de conocimientos integrados e interdisciplinarios, y (v) forma al alumnado para la vida real generando responsabilidad para asumir sus retos.

En los últimos años, se han llevado a cabo numerosos proyectos de innovación docente en las aulas universitarias en los que se han utilizado sistemas de evaluación del aprendizaje basado en problemas. Sin embargo, estos proyectos de innovación han estado principalmente centrados en la incidencia en el aprendizaje (i.e. Fidalgo, Sein-Echaluce, Lerís, y García-Peñalvo, 2013) y son pocos los estudios en el contexto educativo superior que han tenido en cuenta las percepciones del alumnado sobre la justicia del método de evaluación (Niessen, Meijer, y Tendeiro, 2017). En línea con la demanda de procesos de evaluación justos y que conecten al alumnado con las competencias para su desempeño laboral futuro, en esta investigación partimos de la perspectiva de justicia organizacional de Gilliland (1993) para analizar si la evaluación del aprendizaje basado en problemas supone realmente una mejora de la percepción de justicia procedimental frente a la evaluación mediante examen, así como una mejora para el desarrollo de las competencias por parte del alumnado.

\section{CONTEXTO}

El objetivo general del presente estudio comprende la innovación en el ámbito de la metodología docente al poner el foco de atención sobre la posible mejora de la evaluación del aprendizaje basado en problemas frente a la evaluación mediante examen final.

Según la teoría de justicia organizacional de Gilliland (1993), existen una serie de reglas que los métodos de evaluación deben cumplir para ser considerados justos. Algunas de estas reglas son: relación con el puesto, oportunidad para demostrar las competencias para el puesto, oportunidad para reconsiderar los resultados, consistencia en la aplicación de las pruebas, explicación de los resultados, información sobre el proceso, honestidad, efectividad en el trato interpersonal de los evaluadores, comunicación bidireccional, pertinencia de las preguntas, y que el proceso no sea invasivo. Dadas las características de la evaluación del aprendizaje basado en problemas se espera encontrar una mayor percepción de justicia procedimental por parte del alumnado para el aprendizaje basado en problemas que para el examen (H1). Además, puesto que la evaluación del aprendizaje basado en problemas no se centra solo en la obtención de una nota final, sino que durante el proceso el alumnado tiene que poner en práctica una serie de competencias, se espera que el alumnado perciba un mayor desarrollo de competencias mediante la evaluación del aprendizaje basado en problemas que mediante el examen (H2). En general y teniendo en cuenta estas dos posibles mejoras de la evaluación del aprendizaje basado en problemas, se espera que la nota final también sea mayor para esta evaluación que para la evaluación mediante examen (H3).

Otra de las hipótesis propuestas es que, puesto que el aprendizaje basado en problemas requiere un mayor grado de participación y colaboración por parte del alumnado, a mayor grado de participación del alumnado obtendrán mejor nota en la evaluación del aprendizaje basado en problemas (H4). Siguiendo este mismo planteamiento, debido a que la evaluación mediante examen implica que el alumnado sea examinado de los conocimientos explicados en clase, se espera que el alumnado que asiste en mayor medida a las clases obtenga una mejor nota en la evaluación mediante examen (H5).

Por último, dos variables que podrían guardar relación con la percepción de justicia procedimental y el desarrollo de las competencias son la experiencia laboral y el estilo de aprendizaje. Siguiendo a Cuevas y de Ibarrola (2013), un fenómeno creciente en los últimos años es la presencia de estudiantes universitarios que compaginan sus estudios con trabajo y también estudiantes de mayor edad que regresa a las aulas, denominados nontraditional students. Estos autores identificaron en su estudio algunas tensiones entre el ámbito académico y el laboral para los nontraditional students, como que el ámbito académico está alejado de la realidad laboral. En concreto, estos estudiantes podrían preferir un aprendizaje más práctico y orientado a la realización de actividades concretas aplicables en el ámbito laboral (Kenner y Weinerman, 2011). Es por ello que proponemos que si el alumnado trabaja o ha trabajado podría preferir un estilo de aprendizaje basado en la experimentación y en la experiencia más que en la conceptualización abstracta y la observación reflexiva (H6). Dado que el estilo de aprendizaje puede influir en el tipo de experiencias de aprendizaje con las que el alumnado está más cómodo (i.e. Ross, Drysdale, y Schulz, 2001), también planteamos que los estilos de aprendizaje experimentación activa y experiencia concreta correlacionarán de forma directa con la percepción de justicia y la valoración del método de evaluación del aprendizaje basado en problemas como adecuado para el desarrollo de competencias, mientras que los estilos conceptualización abstracta y observación reflexiva lo harán con el método de evaluación mediante examen (H7).

Con la finalidad de poner a prueba estas hipótesis, este estudio se desarrolló como parte de un proyecto de innovación docente en la Universidad de Oviedo. Por su carácter práctico y aplicado, se decidió desarrollar el proyecto en la parte práctica de la asignatura Ergonomía y Riesgos Psicosociales, impartida en el tercer curso del Grado en Relaciones Laborales y Recursos Humanos.

\section{DESCRIPCIÓN}

Para el desarrollo del estudio, 44 estudiantes universitarios, de los cuales $61,4 \%$ eran mujeres y un 45,5\% tenían experiencia laboral, participaron en seis actividades prácticas. Tres de estas actividades se evaluaron mediante examen final con un cuestionario tipo test de dos alternativas de respuesta. Las otras tres prácticas se evaluaron mediante la evaluación del aprendizaje basado en problemas a través de la entrega de informes escritos y exposiciones orales que debían realizar en grupos colaborativos. El alumnado podía obtener un máximo de dos puntos en cada método de evaluación. 
El material didáctico utilizado para el desarrollo del proyecto fue elaborado por los miembros del equipo de investigación y comprendía: (i) la documentación para el desarrollo de las sesiones prácticas, diferenciando entre las sesiones que se evaluaron mediante el examen final y aquellas que se evaluaron siguiendo la metodología del aprendizaje basado en problemas; (ii) el protocolo de evaluación según las dos metodologías de evaluación; y (iii), el instrumento de medida que se aplicó posteriormente a las evaluaciones de las prácticas y que consistía en un cuestionario que contenía las siguientes medidas, además de seis ítems referidos al grado de asistencia y participación en las prácticas evaluadas y a cuestiones sociodemográficas:

- Inventario del estilo de aprendizaje (Kolb, 1985). Este inventario permite identificar el estilo de aprendizaje del alumnado, siendo los estilos: experiencia concreta, experimentación activa, conceptualización abstracta, y observación reflexiva.

- Escala de percepción de justicia procedimental del método de evaluación, adaptada al contexto educativo a partir de la Escala de justicia procedimental en selección (Bauer et a., 2001) que Osca y García (2004) tradujeron y redujeron a 32 ítems. Esta escala mide la justicia procedimental percibida por el alumnado para cada método de evaluación a través de la percepción de: la relación con el puesto de trabajo futuro, la información sobre el proceso, la oportunidad para el desempeño, la oportunidad de reconsiderar los resultados, la consistencia y adecuación, la apertura, el trato recibido, y la comunicación bidireccional. Todos los ítems se midieron en escala Likert.

- Escala de percepción de desarrollo de competencias del método de evaluación. Esta escala fue desarrollada por el equipo de investigación a partir de las competencias incluidas en la guía docente de la asignatura en la que se desarrolló el proyecto. La escala mide en qué grado el alumnado percibe que el proceso de evaluación de cada método le facilita el desarrollo de las competencias. La escala consta de 16 ítems referidos a las siguientes competencias: resolución de problemas; análisis; síntesis; trabajo en equipo; aplicación del conocimiento de salud laboral y prevención de riesgos laborales; transmisión y comunicación por escrito y oralmente usando la terminología propia de las relaciones laborales y los recursos humanos; capacidad para realizar análisis y diagnósticos, prestar apoyo y tomar decisiones en materia de estructura organizativa, organización del trabajo, estudios de métodos y estudios de tiempos de trabajo; capacidad para aplicar técnicas cuantitativas y cualitativas de investigación social al ámbito laboral; capacidad de planificación, diseño, asesoramiento y gestión de los sistemas de prevención de riesgos laborales; y capacidad para interrelacionar las distintas disciplinas que configuran las relaciones laborales. Todos los ítems se midieron en escala Likert.

Para los análisis se utilizó el programa SPSS versión 22. Para el cálculo del tamaño del efecto se utilizó la calculadora desarrollada por Ellis (2009). Se llevaron a cabo comparaciones de medias de muestras relacionadas mediante la prueba $\mathrm{T}$ de Student y se analizaron las correlaciones bivariadas de Pearson entre las variables de interés.
Respecto a los resultados, el alumnado puntuó significativamente más alto $(t=4,11, p=0,00, d=0,32)$ en la escala de percepción de justicia procedimental referida al método de evaluación del aprendizaje basado en problemas ( $M$ $=180,91, D T=26,28)$ que en la referida a la evaluación mediante examen $(M=172,45, D T=26,61)$. Analizando por pares las dimensiones de la escala de justicia procedimental, en todos los casos se encontró una percepción de justicia procedimental significativamente mayor para el método de evaluación del aprendizaje basado en problemas, excepto para la dimensión adecuación y consistencia, para la cual se encontró una mayor percepción de justicia en el caso de la evaluación mediante examen, y para trato recibido y oportunidad de reconsideración de los resultados, para las que no hubo diferencias estadísticamente significativas entre ambos métodos de evaluación.

En el caso de la percepción de adquisición de las competencias, los resultados indicaron nuevamente que el alumnado puntuó en general significativamente más alto ( $t=$ $5,29, p=0,00, d=0,82$ ) en la escala referida al método de evaluación del aprendizaje basado en problemas $(M=62,43$, $D T=8,87)$ que la referida a la evaluación mediante examen $(M=54,70 D T=9,94)$. Analizando por pares cada competencia se encontró una percepción de adquisición de la competencia significativamente mayor para el método de evaluación del aprendizaje basado en problemas que para el examen para todas las competencias excepto para las competencias síntesis, aplicación del conocimiento de salud laboral y prevención de riesgos laborales y gestión de los sistemas de prevención de riesgos laborales, para las cuales no hubo diferencias estadísticamente significativas.

Respecto a la nota obtenida según cada método de evaluación esta fue significativamente $(t=5,13, p=0,00, d=$ $1,05)$ más alta para la evaluación del aprendizaje basado en problemas $(M=1,53, D T=0,39)$ que para el examen $(M=$ $1,14, D T=0,35)$. La Tabla 1 recoge los estadísticos descriptivos de las variables analizadas y la Tabla 2 recoge un resumen de los resultados de los contrastes de hipótesis realizados.

El análisis de correlaciones permitió establecer que el grado de participación en las tres prácticas evaluadas mediante la evaluación del aprendizaje basado en problemas correlacionó de forma significativa y directa con la nota obtenida mediante dicho método (participación práctica $1 r=0,598, p=0,00$; participación práctica $2 r=0,580, p=0,000$; participación práctica $3 r=0,568, p=0,000)$. Cabe destacar también que las competencias que correlacionaron de forma significativa y directa con la participación en las prácticas fueron: resolución de problemas, trabajo en equipo, capacidad para realizar análisis y diagnósticos, prestar apoyo y tomar decisiones, y capacidad de planificación, diseño y asesoramiento de los sistemas de prevención de riesgos laborales. Sin embargo, no se encontró correlación significativa entre la asistencia y la nota obtenida mediante examen. Tampoco se encontró relación entre los estilos de aprendizaje y la percepción de justicia procedimental y la adquisición de las competencias. No obstante, si se encontró correlación significativa entre el hecho de que el alumnado trabaje o haya trabajado y una mayor puntuación en el estilo de experimentación activa ( $r=$ 0,384, $p=0,021)$.

\section{RESUltados}




\section{CONCLUSIONES}

Tabla 1

Estadísticos descriptivos para la escala de justicia procedimental

\begin{tabular}{lccrr}
\hline \multicolumn{1}{c}{ Variable } & Media & Máximo & $\begin{array}{r}\text { Desv. } \\
\text { típica }\end{array}$ & $\alpha$ \\
\hline Justicia procedimental AP & 180,91 & \multirow{2}{*}{224} & 26,28 & 0,95 \\
Justicia procedimental EX & 172,45 & & 26,61 & 0,94 \\
Competencias AP & 62,43 & \multirow{2}{*}{80} & 8,87 & 0,91 \\
Competencias EX & 54,70 & & 9,94 & 0,91 \\
Nota AP & 1,53 & 2 & 0,39 & - \\
Nota EX & 1,14 & 2 & 0,35 & - \\
Experiencia Concreta & 14,27 & & 3,41 & - \\
Observación Reflexiva & 16,32 & \multirow{2}{*}{24} & 3,84 & - \\
Conceptualizacion Abstracta & 16,00 & & 2,86 & - \\
Experimentacion Activa & 14,95 & & 3,18 & - \\
Asistencia práctica 1 & 4,30 & & 1,13 & - \\
Asistencia práctica 2 & 4,20 & & 1,19 & - \\
Asistencia práctica 3 & 4,50 & \multirow{2}{*}{5} & 0,95 & - \\
Participación práctica 4 & 4,18 & & 1,00 & - \\
Participación práctica 5 & 4,20 & & 1,00 & - \\
Participación práctica 6 & 4,02 & & 1,19 & - \\
\hline
\end{tabular}

Nota: AP = Evaluación del aprendizaje basado en problemas. EX = Evaluación mediante examen.

Tabla 2

Contrastes de hipótesis para la comparación de medias entre métodos

\begin{tabular}{|c|c|c|}
\hline Comparación de medias entre métodos & $\begin{array}{c}\mathrm{T} \text { de } \\
\text { Student }\end{array}$ & $p$ \\
\hline Justicia procedimental & 4,11 & 0,00 \\
\hline Relación con el puesto & 5,28 & 0,00 \\
\hline Información sobre el proceso & 4,98 & 0,00 \\
\hline Oportunidad desempeño & 4,23 & 0,00 \\
\hline Oportunidad reconsideración & 1,07 & 0,29 \\
\hline Consistencia y adecuación, & 2,77 & 0,00 \\
\hline Apertura & 2,80 & 0,00 \\
\hline Trato recibido & 1,63 & 0,11 \\
\hline Comunicación bidireccional & 2,43 & 0,02 \\
\hline Competencias & 5,29 & 0,00 \\
\hline Resolución de problemas & 2,63 & 0,01 \\
\hline Análisis & 2,79 & 0,01 \\
\hline Síntesis & 0,141 & 0,88 \\
\hline Trabajo en equipo & 5,42 & 0,00 \\
\hline Aplicación del conocimiento & 1,95 & 0,58 \\
\hline Comunicación escrita & 3,13 & 0,00 \\
\hline Comunicación oral & 4,27 & 0,00 \\
\hline Análisis y diagnósticos & 3,35 & 0,00 \\
\hline Prestar apoyo & 2,55 & 0,02 \\
\hline Tomar decisiones & 2,23 & 0,031 \\
\hline Aplicar técnicas cuantitativas & 3,53 & 0,00 \\
\hline Aplicar técnicas cualitativas & 3,46 & 0,00 \\
\hline Planificación y diseño & 4,79 & 0,00 \\
\hline Asesoramiento & 2,71 & 0,01 \\
\hline Gestión sistemas & 1,61 & 0,12 \\
\hline Interrelacionar disciplinas & 2,30 & 0,026 \\
\hline (2) & 5,13 & 0,00 \\
\hline
\end{tabular}

\section{Relación con el puesto}

Oportunidad desempeño

Apertura

Trato recibido

encias

Análisis

Trabajo en equipo

Aplicación del conocimiento

Comunicación oral

Análisis y diagnósticos

Tomar decisiones

Planificación y diseño
En este estudio analizamos las diferencias en cuanto a la percepción de justicia procedimental y desarrollo de las competencias entre el método de evaluación del aprendizaje basado en problemas y el examen. Mediante la comparación de medias se encontró que, en general, la evaluación del aprendizaje basado en problemas fue mejor percibida en cuanto a justicia procedimental que el examen. Cabe destacar no obstante que la dimensión adecuación y consistencia obtuvo mayor puntuación para el examen. Siguiendo la teoría de justicia de Gilliland (1993), ello puede deberse a que la evaluación de las preguntas del examen seguía una plantilla de corrección que podría ser percibida por el alumnado como libre de sesgos, mientras que la evaluación del aprendizaje basado en problemas seguía un protocolo de evaluación que podría ser percibido como más subjetivo.

Además, en este estudio también se encontró que la evaluación del aprendizaje basado en problemas fue mejor percibida en general que el examen como un método para desarrollar las competencias establecidas en la guía docente de la asignatura. No obstante, analizando para qué competencias se producían las diferencias se encontró que la evaluación del aprendizaje basado en problemas solo suponía una mejora frente al examen para las siguientes competencias: resolución de problemas; análisis; aplicación del conocimiento de salud laboral y prevención de riesgos laborales; capacidad para realizar análisis y diagnósticos, prestar apoyo y tomar decisiones en materia de estructura organizativa, organización del trabajo, estudios de métodos y estudios de tiempos de trabajo; capacidad de planificación, diseño, asesoramiento de los sistemas de prevención de riesgos laborales; y capacidad para interrelacionar las distintas disciplinas que configuran las relaciones laborales. Para las competencias restantes no se encontraron diferencias estadísticamente significativas entre ambos métodos. Esto nos lleva a pensar que la mejora significativa en la percepción del desarrollo de algunas de las competencias es beneficiosa para el alumnado y está en la línea con las demandas de un enfoque basado en competencias. Sin embargo, debe tenerse en cuenta que esta mejora no ha sido significativa para todas las competencias, por lo que es importante valorar si el desarrollo de la metodología de evaluación mediante aprendizaje basado en problemas puede realmente suponer una mejora para la evaluación según cada caso particular y las competencias que se quieran desarrollar. A pesar de ello, debe destacarse que los resultados mostraron que la nota final fue significativamente mejor en el caso de la evaluación del aprendizaje basado en problemas que en el caso de la evaluación mediante examen.

Otro de los resultados encontrados en este estudio fue que la participación del alumnado correlacionó con la nota obtenida mediante la evaluación del aprendizaje basado en problemas, mientras que no se encontró relación entre la asistencia a las clases y la nota del examen. Esto pone de relevancia que la implicación del alumnado para la evaluación del aprendizaje basado en problemas es importante y está en línea con la demanda del Espacio Europeo de Educación Superior de situar al alumnado como foco central del proceso de aprendizaje (Delgado y Oliver, 2006).

También cabe destacar que el alumnado con experiencia laboral poseía un estilo de aprendizaje más práctico, pero ninguno de los estilos de aprendizaje correlacionó de forma específica con la percepción de justicia y de competencias de 
alguno de los métodos. Esto sugiere que la evaluación del aprendizaje basado en problemas podría ser implantada independientemente del estilo de aprendizaje del alumnado.

Como principales limitaciones del estudio debe mencionarse, por un lado, que se realizó en la parte práctica de una asignatura que suponía solo el $40 \%$ de la nota final, por lo que el alumnado podría no estar igual de implicado que en el caso de que la nota a obtener fue más relevante para la nota final de la asignatura. Por otro lado, el estudio se ha realizado en una sola asignatura, por lo que para generalizar los resultados se debería llevar a cabo con una muestra más amplia de diferentes asignaturas y titulaciones, así como ampliar los métodos de evaluación utilizados para poder hacer una comparación más exhaustiva.

\section{AGRADECIMIENTOS}

Esta investigación ha sido posible gracias al proyecto de investigación con ref. PSI-2013-44854-R (Ministerio de Economía y Competitividad y Fondos Sociales Europeos)

\section{REFERENCIAS}

Barrows, H. S. (1986). A Taxonomy of problem-based learning methods. Medical Education, 20(6), 481-486. doi: http://doi.org/10.1111/j.1365-2923.1986.tb01386.X

Bauer, T. N., Truxillo, D. M., Sanchez, R. J., Craig, J. M., Ferrara, P., y Campion, M. A. (2001). Applicant reactions to selection: Development of the selection procedural justice scale (SPJS). Personnel Psychology, 54(2), pp. 388-420.

Cohen-Charash, Y., y Spector, P. E. (2001). The role of justice in organizations: a metaanalysis. Organizational Behavior and human Decision Processes, 86, 278-321. doi: https://doi.org/10.1006/obhd.2001.2958

Coll, C., Rochera, M. J., Mayordomo, R. M., y Naranjo, M. (2007). Evaluación continua y ayuda al aprendizaje. Análisis de una experiencia de innovación en educación superior con apoyo de las TIC. Revista Electrónica de Investigación Psicoeducativa, 13(5), 783-804. Recuperado el 23 de agosto de 2016 de http://www.investigacionpsicopedagogica.org/revista/articulos/13/espannol/Art_1 3_205.pdf

Colquitt, J. A., Conlon, D. E., Wesson, M. J., Porter, C. O., y Ng, K. Y. (2001). Justice at the millennium: a metaanalytic review of 25 years of organizational justice research. Journal of Applied Psychology, 86(3), 425-445. doi: http://dx.doi.org/10.1037/0021-9010.86.3.425

Cuevas, J. F., y de Ibarrola, M. (2013). Vidas cruzadas. Los estudiantes que trabajan: un análisis de sus aprendizajes. Revista de la educación superior, 42(165), 124-148. Recuperado el 23 de agosto de 2016 de http://www.scielo.org.mx/scielo.php?pid=S018527602013000100007\&script=sci_arttext

Delgado, A. M., y Oliver, R. (2006). La evaluación continua en un nuevo escenario docente. Revista de Universidad y Sociedad del Conocimiento, 3(1), 1-13. Recuperado el 23 de agosto de 2016 de http://www.uoc.edu/rusc/3/1/dt/esp/delgado_oliver.pdf
Ellis, P. D. (2009). Effect size calculators. Acceso el 4 de mayo de 2017 en http://www.polyu.edu.hk/mm/effectsizefaqs/calculator/c alculator.html

Fidalgo, A., Sein-Echaluce, M. L., Lerís, D., y GarcíaPeñalvo, F. J. (2013). Sistema de Gestión de Conocimiento para la aplicación de experiencias de innovación educativa en la formación. En Á. Fidalgo, y $\mathrm{M}^{\mathrm{a}}$ L. Sein-Echaluce (Eds.), Actas del II Congreso Internacional sobre Aprendizaje, Innovación y Competitividad, CINAIC 2013, Madrid, 6-8 de noviembre de 2013 (pp. 750-755). Madrid, España: Fundación General de la Universidad Politécnica de Madrid. Recuperado el 4 de mayo de 2017 de https://gredos.usal.es/jspui/handle/10366/122586

Gil, J., y Padilla, M. T. (2009). La participación del alumnado universitario en la evaluación del aprendizaje. Educación $X X 1,12$, 43-65. Recuperado el 23 de agosto de 2016 de http://www.redalyc.org/pdf/706/70611919004.pdf

Gilliland, S. W. (1993). The perceived fairness of selection systems: An organizational Justice perspective. Administrative of Management Review, 18, 694-734.

Kenner, C., y Weinerman, J. (2011). Adult learning theory: Applications to non-traditional college students. Journal of College Reading and Learning, 41(2), 87-96. doi: http://dx.doi.org/10.1080/10790195.2011.10850344

Kolb, D. A. (1985). Learning Style Inventory, Revised Edition. Boston, MA: McBer \& Company.

Niessen, A. S. M., Meijer, R. R, y J. N. Tendeiro. (2017). Applying organizational justice theory to admission into higher education: Admission from a student perspective. International Journal of Selection and Assessment, 25(1), 72-84. doi: http://doi.org/10.1111/ijsa.12161

Osca, A., y García, L. (2004). ¿Cómo perciben los candidatos los procesos de selección? Una aproximación desde el modelo de Justicia Procedimental de Gilliland (1993). Revista de Psicología del Trabajo y de las Organizaciones, 20(2), 225-247. Recuperado el 4 de mayo de 2017 de http:/www.redalyc.org/html/2313/231317999006/

Ross, J. L., Drysdale, M. T., y Schulz, R. A. (2001). Cognitive learning styles and academic performance in two postsecondary computer application courses. Journal of Research on Computing in Education, 33(4), 400-412. doi: http://dx.doi.org/10.1080/08886504.2001.10782324

Solaz-Portolés, J. J., Sanjosé, V., y Gómez, A. (2011). Aprendizaje basado en problemas en la Educación Superior: una metodología necesaria en la formación del profesorado. Didáctica de las Ciencias Experimentales y Sociales, 25, 177-186. Recuperado el 4 de mayo de 2017 de http://roderic.uv.es/handle/10550/21337

Zaragoza, J., Luis-Pascual, J. C., y Manrique, J. C. (2009). Experiencias de innovación en docencia universitaria: resultados de la aplicación de sistemas de evaluación formativa. Revista de Docencia Universitaria, 4, 1-33. Recuperado el 23 de agosto de 2016 de http://revistas.um.es/redu/article/view/92561/89051 